Journal of Organometallic Chemistry, 241 (1983) 333-342

Elsevier Sequoia S.A., Lausannc - Printed in The Netherlands

\title{
METALLORGANISCHE LEWIS-SÄUREN; METALLKOMPLEXE MIT SCHWACH KOORDINIERTEN LIGANDEN
}

\author{
XI *. ACETYLVERBRÜCKTE KATIONISCHE KOMPLEXE VON \\ MOLYBDÄN, EISEN UND MANGAN; STRUKTUR VON \\ $\left[\left(\pi-\mathrm{C}_{5} \mathrm{H}_{5}\right)(\mathrm{CO})_{2} \mathrm{Mo}\left(\mu_{2}-\eta^{2}-\mathrm{CH}_{3} \mathrm{CO}\right) \mathrm{Mo}(\mathrm{CO})_{2}\left(\pi-\mathrm{C}_{5} \mathrm{H}_{5}\right)\right]^{+} \mathrm{BF}_{4}^{-}$
}

\author{
KARLHEINZ SÜNKEL, KLAUS SCHLOTER, WOLFGANG BECK \\ Institut für Anorganische Chemie der Universität München, Meiserstr. 1, 8000 München 2(B.R.D)
}

KLAUS ACKERMANN und ULRICH SCHUBERT *

Anorganisch-Chemisches Institut der Technischen Universität München, Lichtenbergstr. 4, 8046 Garching (B.R.D.)

(Eingegangen den 20. Juli 1982)

\section{Summary}

The highly reactive complexes $\left(\pi-\mathrm{C}_{5} \mathrm{H}_{5}\right)(\mathrm{CO})_{3} \mathrm{MoX}\left(\mathrm{X}=\mathrm{FBF}_{3}, \mathrm{FSbF}_{5}\right)$ react with $\left(\pi-\mathrm{C}_{5} \mathrm{H}_{5}\right)(\mathrm{CO})_{3} \mathrm{MoCH}_{3},\left(\pi-\mathrm{C}_{5} \mathrm{H}_{5}\right)(\mathrm{CO})_{2} \mathrm{FeCH}_{3}$ or $\left(\pi-\mathrm{C}_{5} \mathrm{H}_{5}\right)(\mathrm{CO})_{2} \mathrm{FeCOCH}_{3}$ and cis-, trans- $\mathrm{Ph}_{3} \mathrm{P}(\mathrm{CO})_{4} \mathrm{MnCH}_{3}$ to give $\left[\left(\pi-\mathrm{C}_{5} \mathrm{H}_{5}\right)(\mathrm{CO})_{2} \mathrm{Mo}\left(\mu_{2}-\eta^{2}-\mathrm{CH}_{3} \mathrm{CO}\right)-\right.$ $\left.\mathrm{Mo}(\mathrm{CO})_{2}\left(\pi-\mathrm{C}_{5} \mathrm{H}_{5}\right)\right]^{+} \mathrm{BF}_{4}^{-}(\mathrm{I}),\left[\left(\pi-\mathrm{C}_{5} \mathrm{H}_{5}\right)(\mathrm{CO})_{2} \mathrm{Fe}-\mathrm{C}\left(\mathrm{CH}_{3}\right) \mathrm{O}-\mathrm{Mo}(\mathrm{CO})_{3}\left(\pi-\mathrm{C}_{5} \mathrm{H}_{5}\right)\right]^{+}$ $\mathrm{SbF}_{6}^{-}$(II) and $\left[\left(\mathrm{Ph}_{3} \mathrm{P}\right)(\mathrm{CO})_{4} \mathrm{Mn}-\mathrm{C}\left(\mathrm{CH}_{3}\right) \mathrm{O}-\mathrm{Mo}(\mathrm{CO})_{3}\left(\pi-\mathrm{C}_{5} \mathrm{H}_{5}\right)\right]^{+} \mathrm{BF}_{4}^{-}$(II), respectively. The $\mathrm{X}$-ray structure of $\mathrm{I}$ has been determined. It shows a symmetrical acetyl bridge of the type<smiles>CC12OOC1(C)C1(CO1)O2</smiles>

\section{Zusammenfassung}

Die Tetrafluoroborato- und Hexafluoroantimonato-Komplexe $\left(\pi-\mathrm{C}_{5} \mathrm{H}_{5}\right)$ $(\mathrm{CO})_{3} \mathrm{MoX}\left(\mathrm{X}=\mathrm{FBF}_{3}, \mathrm{FSbF}_{5}\right)$ setzen sich mit $\left(\pi-\mathrm{C}_{5} \mathrm{H}_{5}\right)(\mathrm{CO})_{3} \mathrm{MoCH}_{3},\left(\pi-\mathrm{C}_{5} \mathrm{H}_{5}\right)-$ $(\mathrm{CO})_{2} \mathrm{FeCH}_{3}$ oder $\left(\pi-\mathrm{C}_{5} \mathrm{H}_{5}\right)(\mathrm{CO})_{2} \mathrm{FeCOCH}_{3}$ und cis-, trans- $\mathrm{Ph}_{3} \mathrm{P}(\mathrm{CO})_{4^{-}}$

\footnotetext{
* X. Mitteilung siehe Ref. 1 .
} 
$\mathrm{MnCOCH}_{3}$ zu $\left[\left(\pi-\mathrm{C}_{5} \mathrm{H}_{5}\right)(\mathrm{CO})_{2} \mathrm{Mo}\left(\mu_{2}-\eta^{2}-\mathrm{COCH}_{3}\right) \mathrm{Mo}(\mathrm{CO})_{2}\left(\pi-\mathrm{C}_{5} \mathrm{H}_{5}\right)\right]^{i \cdot} \mathrm{BF}_{4}{ }^{-}$(I), $\left[\left(\pi-\mathrm{C}_{5} \mathrm{H}_{5}\right)(\mathrm{CO})_{2} \mathrm{Fe}-\mathrm{C}\left(\mathrm{CH}_{3}\right) \mathrm{O}-\mathrm{Mo}(\mathrm{CO})_{3}\left(\pi-\mathrm{C}_{5} \mathrm{H}_{5}\right)\right]^{+} \mathrm{SbF}_{6} \quad$ (II) und $\left[\left(\mathrm{Ph}_{3} \mathrm{P}\right)-\right.$ $\left.(\mathrm{CO})_{4} \mathrm{Mn}-\mathrm{C}\left(\mathrm{CH}_{3}\right) \mathrm{O}-\mathrm{Mo}(\mathrm{CO})_{3}\left(\pi-\mathrm{C}_{5} \mathrm{H}_{5}\right)\right]^{+} \mathrm{BF}_{4}{ }^{-\infty}$ (III) um. Die Röntgenstrukturanalyse von I zeigt eine symmetrische Acetylbrücke des Typs

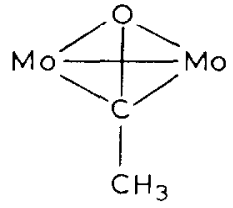

Durch Umsetzung der hochreaktiven Tetrafluoroboratkomplexe $\left(\pi-\mathrm{C}_{5} \mathrm{H}_{5}\right)(\mathrm{CO})_{3^{-}}$ $\mathrm{MFBF}_{3}(\mathrm{M}=\mathrm{Mo}$, W) mit Hydrido-Komplexen lassen sich gezielt hydridverbrückte Verbindungen darstellen [2,3]. Allgemein sind solche Komplexe aus Metallhydriden und Metall-Komplexen mit schwach koordinierten anionischen (z.B. BF $\quad[2,3]$, $\mathrm{PF}_{6}^{-}$[3]) oder Neutral-Liganden [4] zugänglich. Diese Reaktionen lassen sich als Lewis-Säure-Base-Reaktionen auffassen [4]:

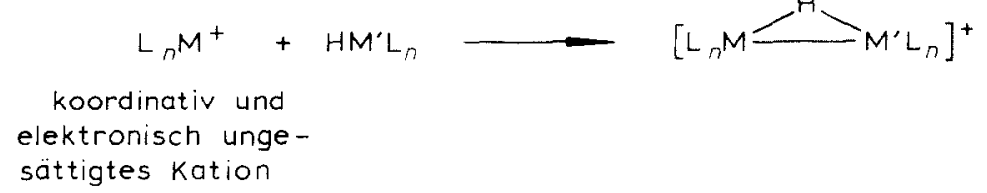

Nach dem gleichen Prinzip werden auch halogenid- und pseudohalogenidverbrückte Komplexe erhalten [5].

Entsprechend diesem Schema versuchten wir durch Umsetzung der $\mathrm{BF}_{4}{ }^{-}$-bzw. $\mathrm{SbF}_{6}{ }^{-}$-Komplexe $\left(\pi-\mathrm{C}_{5} \mathrm{H}_{5}\right)(\mathrm{CO})_{3} \mathrm{MoX} \quad\left(\mathrm{X}=\mathrm{FBF}_{3}, \quad \mathrm{FSbF}_{5}\right)$ mit verschiedenen Metallalkyl-Verbindungen methylverbrückte Komplexe darzustellen [6]. Bei der Reaktion von $\left(\pi-\mathrm{C}_{5} \mathrm{H}_{5}\right)(\mathrm{CO})_{3} \mathrm{MoFBF}_{3}$ mit $\left(\pi-\mathrm{C}_{5} \mathrm{II}_{5}\right)(\mathrm{CO})_{3} \mathrm{MoCII}_{3}$ entsteht jedoch der symmetrisch acetylverbrückte Komplex I

$[\left(\pi-\mathrm{C}_{5} \mathrm{H}_{5}\right)(\mathrm{CO})_{2} \mathrm{MO}-\overbrace{\mathrm{O}}^{\mathrm{C}} \mathrm{MO}(\mathrm{CO})_{2}\left(\pi-\mathrm{C}_{5} \mathrm{H}_{5}\right)]^{+} \mathrm{BF}_{4}$

(1)

Die Struktur dieser Verbindung mit einer $\mu_{2}-\eta^{2}-\mathrm{CH}_{3} \mathrm{CO}-\mathrm{Gruppe}$ wurde röntgenographisch gesichert (vgl. unten). LaCroce und Cutler konnten I vor kurzem auf die gleiche Weise als $\mathrm{PF}_{6}-\mathrm{Salz}$ erhalten und aufgrund spektroskopischer Daten die richtige Struktur ableiten [7]. Da I in Lösung scharfe ${ }^{1} \mathrm{H}-\mathrm{NMR}$-Signale zeigt, ist es diamagnetisch. Daher muss in I eine Mo-Mo-Wechselwirkung vorliegen. Bei unseren Reaktionen erhielten wir keinen Hinweis auf die Bildung eines $\mu$-AcetylKomplexes $\left[\left(\pi-\mathrm{C}_{5} \mathrm{H}_{5}\right)(\mathrm{CO})_{3} \mathrm{Mo}-\mathrm{C}\left(\mathrm{CH}_{3}\right) \mathrm{O}-\mathrm{Mo}(\mathrm{CO})_{3}\left(\pi-\mathrm{C}_{5} \mathrm{H}_{5}\right)\right]^{+}$[7]. I entsteht auch direkt aus der Reaktion von $\left(\pi \cdot \mathrm{C}_{5} \mathrm{H}_{5}\right)(\mathrm{CO})_{3} \mathrm{MoCH} \mathrm{H}_{3}$ mit $\mathrm{Ph}_{3} \mathrm{CBF}_{4}$, wobei man annehmen muss, dass sich zunächst durch Methylabstraktion das Kation "( $\pi$ - 
$\left.\mathrm{C}_{5} \mathrm{H}_{5}\right)(\mathrm{CO})_{3} \mathrm{Mo}^{+}$" bildet, das sich mit weiterem Methyl-Komplex zu I umsetzt. Die Bildung von I lässt sich leicht verstehen; durch elektrophilen Angriff der Lewis-Säure $\left(\pi-\mathrm{C}_{5} \mathrm{H}_{5}\right)(\mathrm{CO})_{3} \mathrm{Mo}^{+}$am Sauerstoffatom eines CO-Liganden wird dic Methyl-

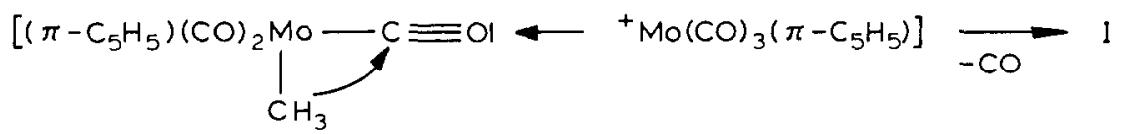

wanderung 7um nun "positivierten" C-Atom des CO-Liganden erleichtert. Über die Aktivierung der Methylwanderung in Carbonyl-Komplexen durch Lewissäuren wurde schon früher berichtet [8].

Die $\eta^{\prime}$-acetylverbrückten Komplexe II und III entstehen bei der Umsetzung von $\left(\pi-\mathrm{C}_{5} \mathrm{H}_{5}\right)(\mathrm{CO})_{3} \mathrm{MoX}\left(\mathrm{X}=\mathrm{FBF}_{3}, \mathrm{FSbF}_{5}\right)$ mit $\left(\pi-\mathrm{C}_{5} \mathrm{H}_{5}\right)(\mathrm{CO})_{2} \mathrm{FeCH}_{3}$ oder $(\pi-$ $\left.\mathrm{C}_{5} \mathrm{H}_{5}\right)(\mathrm{CO})_{2} \mathrm{FeCOCH}_{3}$ bzw. cis-, trans- $\left(\mathrm{Ph}_{3} \mathrm{P}\right)(\mathrm{CO})_{4} \mathrm{MnCOCH}_{3}$.

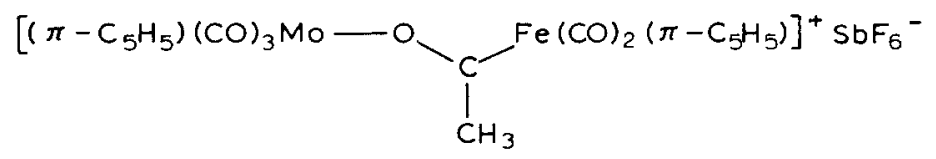

(II)

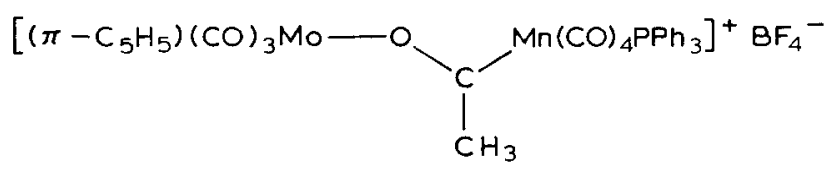

(III)

Die Struktur von II, das vor kurzem als $\mathrm{PF}_{6}{ }^{-}$-Salz erhalten wurde [7], konnte inzwischen durch eine Röntgenstrukturanalyse bestätigt werden [9].

Charakteristische IR- und NMR-Daten von I-III sind in Tab. 1 zusammengefasst. Die CO-Bande der Acylbrücke von II und III liegt, wie zu erwarten, bei sehr kleinen Wellenzahlen $\left(\sim 1480 \mathrm{~cm}^{-1}\right)$. Für I wurde keine charakteristische CO-Absorption der Acylbrücke im IR-Spektrum gefunden. Verschiedene $\eta^{1}$-acylverbrückte Komplexe wurden bereits früher beschrieben [10]. Eine Acylbrücke wie in I konnte

TABELLE 1

SPEKTROSKOPISCHE DATEN DER KOMPLEXE I-III

\begin{tabular}{|c|c|c|c|c|}
\hline \multirow[t]{2}{*}{ Verbindung } & \multicolumn{2}{|l|}{ IR } & \multicolumn{2}{|c|}{${ }^{1} \mathrm{H}-\mathrm{NMR}$ (in $\mathrm{CD}_{2} \mathrm{Cl}_{2}$ ) } \\
\hline & $\begin{array}{l}\nu(\mathrm{CO}) \\
\left(\mathrm{cm}^{-1}\right)\end{array}$ & $\begin{array}{l}\nu(\mathrm{C}=0) \\
\left(\mathrm{cm}^{-1}\right)\end{array}$ & $\begin{array}{l}\delta\left(\mathrm{C}_{5} H_{5}\right) \\
(\mathrm{ppm})\end{array}$ & $\begin{array}{l}\delta\left(\mathrm{CH}_{3} \mathrm{CO}\right) \\
(\mathrm{ppm})\end{array}$ \\
\hline I & $\begin{array}{l}2080 \mathrm{~s}, 2060 \mathrm{vs}, 2040 \mathrm{vs}, \\
2020 \mathrm{vs}, 1995 \mathrm{vs}, 1940 \mathrm{vs} \\
1875 \mathrm{~s} \text { (in KBr) }\end{array}$ & & 5.88 & 2.96 \\
\hline II & $\begin{array}{l}\text { 2063vs, } 2049 v s, 1992 v s, \\
1970 v s \text { (in Nujol) }\end{array}$ & 1480 & $6.03,5.03$ & 2.52 \\
\hline III & $2086 \mathrm{~m}, 2062 \mathrm{~s}, 1968 \mathrm{vs}$ (in Nujol) & 1483 & $\begin{array}{l}6.04,5.69 \\
\left(-60^{\circ} \mathrm{C}\right)\end{array}$ & $\begin{array}{l}2.67,2.38 \\
\left(-60^{\circ} \mathrm{C}\right)\end{array}$ \\
\hline
\end{tabular}


bisher nur an einem formyl-verbrückten Komplex strukturell gesichert werden [11].

Über die Ergebnisse der Umsetzungen von $(\mathrm{CO})_{5} \mathrm{M}-\mathrm{CH}_{3}(\mathrm{M}=\mathrm{Mn}$, Re) mit $\left(\pi-\mathrm{C}_{5} \mathrm{H}_{5}\right)(\mathrm{CO})_{3} \mathrm{MOFBF}_{3}$ sowie über die Röntgenstrukturanalyse von II wird an anderer Stelle berichtet.

\section{Beschreibung der Struktur von I}

Die asymmetrische Einheit von I enthält zwei symmetrie-unabhängige Moleküle, deren Strukturparameter jedoch keine signifikanten Unterschiede aufweisen (Tab. 3).

In I werden zwei $\left(\pi-\mathrm{C}_{5} \mathrm{H}_{5}\right)(\mathrm{CO})_{2}$ Mo-Einheiten jeweils "side-on" durch einen Acetyl-Liganden überbrückt, der symmetrisch zwischen beiden MetallkomplexFragmenten lokalisiert ist. Die Abstände der Mo-Atome zu den Brücken-Sauerstoffatomen (Mittel $213(1) \mathrm{pm}$ ) sind nur wentg länger als zu den Brücken-Kohlenstoffatomen (Mittel 209(2) pm); es liegt also echte $\eta^{2}$-Koordination an beide Übergangsmetalle vor. Das zentrale $\mathrm{Mo}_{2} \mathrm{CO}$-Fragment ist längs der $\mathrm{C}$-O-Achse gefaltet: die beiden MoCO-Ebenen schliessen einen Winkel von $86.7^{\circ}$ ein. die beiden Mo-Atome nähern sich auf $291.0 \mathrm{pm}$.

In dem zu 1 isoelektronischen Komplex $\left(\pi-\mathrm{C}_{5} \mathrm{H}_{3}\right)(\mathrm{OC})_{2} \mathrm{Mo}\left(\mu-\eta^{2}-\mathrm{CNPh}\right)-$ $\mathrm{Mo}(\mathrm{CO})_{2}\left(\pi-\mathrm{C}_{5} \mathrm{H}_{5}\right)$ [12] wurde eine nicht symmetrisch angeordnete $\boldsymbol{\sigma}-\pi$-Isocyanidbrücke zwischen den beiden Mo-Atomen gefunden, wobei der Mo-Mo-Abstand mit 323.5 pm grösser ist als in $I$.

Durch die Koordination an die Metalle sollte sich der C O-Abstand der AcylEinheit verlängern. Obwohl wegen der hohen Standardabweichungen eine detailliertere Diskussion nicht sinnvoll ist, zeigt sich jedoch, dass eine starke Aufweitung, wie sie mit $150(1) \mathrm{pm}$ im analog gebauten Formyl-Komplex $\left[\left(\eta^{5}-\mathrm{C}_{5} \mathrm{Me}_{4} \mathrm{Et}\right) \mathrm{TaCl}_{2}\right]_{2-}$ $(\mu-\mathrm{H})(\mu-\mathrm{CHO})[11]$ heobachtet wird, in I bei weitem nicht erreicht wird. Jedes der beiden $\left(\pi-\mathrm{C}_{5} \mathrm{H}_{5}\right)(\mathrm{CO})_{2} \mathrm{Mo}$-Fragmente besitzt die in pseudooktaedrischen Komplexen der Art $\left(\pi-\mathrm{C}_{5} \mathrm{H}_{5}\right)(\mathrm{CO})_{2} \mathrm{ML}(\mathrm{M}=$ Übergangsmetall) zu erwartende Geometrie. Das in diesen Komplexen auf $\mathrm{L}$ gerichtete Orbital zeigt bei $\mathrm{I}$ etwa ins Zentrum des jeweiligen (Nachbar-)Mo,C.O-Dreiecks. Die rotamere Einstellung des Acyl-Liganden relativ $\mathrm{zu}$ den $\left(\pi-\mathrm{C}_{5} \mathrm{H}_{5}\right)(\mathrm{CO})_{2}$ Mo-Resten ist dagegen in den beiden Molekülhälften von I verschieden (s. Fig. 1). Während an $\operatorname{Mo}(2)$ (analog an $\operatorname{Mo}(3)$ ) die Winkel $\mathrm{Mo}(1)-\mathrm{Mo}(2)-\mathrm{C}(\mathrm{CO})$ bzw. $\mathrm{C}(10)(\mathrm{O}(10))-\mathrm{Mo}(2)-\mathrm{C}(\mathrm{CO})$ symmetrisch und der Schwerpunkt des $\left(\pi-\mathrm{C}_{5} \mathrm{H}_{5}\right)$-Rings und Mo(1) ungefähr transständig zueinander sind, ist der $\left(\pi-\mathrm{C}_{5} \mathrm{H}_{5}\right)(\mathrm{CO})_{2}$ Mo-Rest mit $\mathrm{Mo}(1)$ als Zentralatom relativ zum Acyl-Liganden so gedreht, dass die beiden $\mathrm{C}(10)-\mathrm{Mo}(1)-\mathrm{C}(\mathrm{CO})$-Winkel ungefähr gleich werden und der Schwerpunkt des $\left(\pi-\mathrm{C}_{5} \mathrm{H}_{5}\right)$-Ringes ungefähr trans zu C(10) ist. Im Formyl-Komplex [( $\left.\left.\eta^{5}-\mathrm{C}_{5} \mathrm{Me}_{4} \mathrm{Et}\right) \mathrm{TaCl}_{2}\right]_{2}(\mu-\mathrm{H})(\mu-\mathrm{CHO})$ [11] findet sich eine vollkommen äquivalente Anordnung der $\left(\pi-\mathrm{C}_{5} \mathrm{H}_{5}\right) \mathrm{TaCl}_{2}$-Einheiten relativ zum Formyl-Liganden (mit dem Unterschied, dass die Positionen von Kohlenstoff und Sauerstoff gegenüber I vertauscht sind). MO-Rechnungen haben gezeigt [13], dass $\pi$-Orbitale von "single-faced"-Acceptor-Liganden mit den Metallorbitalen von $\left(\pi-\mathrm{C}_{5} \mathrm{H}_{5}\right)(\mathrm{CO})_{2} \mathrm{M}-$ Fragmenten am besten überlappen können, wenn sie auf der Spiegelebene des $\left(\pi-\mathrm{C}_{5} \mathrm{H}_{5}\right)(\mathrm{CO})_{2} \mathrm{M}$-Fragmentes senkrecht sehen. Der Energieunterschied zu anderen Stellungen ist jedoch klein genug. um z.B. durch sterische Erfordernisse überwunden werden zu können (vgl. [14]). Man kann dạvon ausgehen, dass in $\mathrm{I}$ eine relative Verdrehung der beiden $\left(\pi-\mathrm{C}_{5} \mathrm{H}_{5}\right)(\mathrm{CO})_{2} \mathrm{Mo}$-Einheiten 

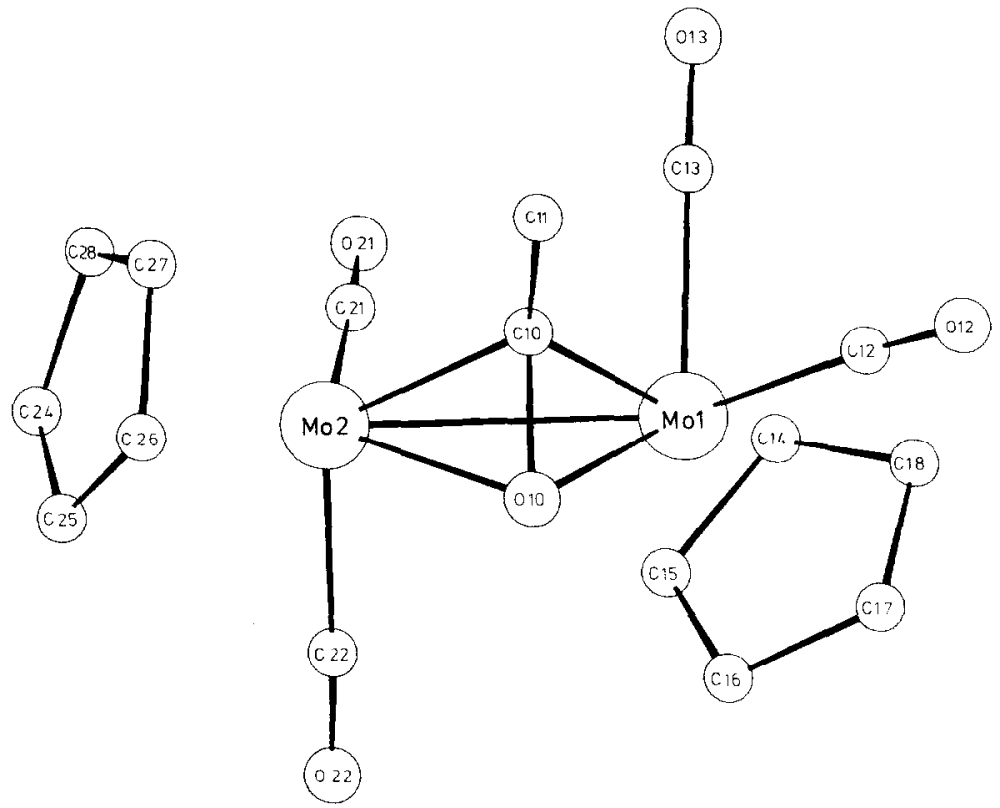

Fig. 1. Röntgestrukturanalyse von Komplex I.

gegeneinander einer Verminderung sterischer Wechselwirkungen zwischen beiden Metallkomplex-Fragmenten dient.

\section{Experimenteller Teil}

Für die Handhabung der empfindlichen metallorganischen Lewissäuren verweisen wir auf frühere Arbeiten [2]. $\left(\mathrm{C}_{5} \mathrm{H}_{5}\right) \mathrm{Mo}(\mathrm{CO})_{3} \mathrm{CH}_{3}$ [15a], $\left(\mathrm{C}_{5} \mathrm{H}_{5}\right) \mathrm{Fe}(\mathrm{CO})_{2} \mathrm{CH}_{3}$ [15b], $\left(\mathrm{C}_{5} \mathrm{H}_{5}\right) \mathrm{Fe}(\mathrm{CO})_{2} \mathrm{COCH}_{3}$ [16] und $\left(\mathrm{Ph}_{3} \mathrm{P}\right)(\mathrm{CO})_{4} \mathrm{MnCOCH}_{3}$ [17] wurden nach Literaturangaben erhalten.

Für die IR-Messungen stand ein Perkin-Elmer-Gerät Modell 325, für die ${ }^{1} \mathrm{H}$ NMR-Spektren ein JEOL FX-90-NMR-Gerät zur Verfügung.

$\mu_{2}-\eta^{2}$-Acetyl-(tetracarbonyl)(di- $\pi$-cyclopentadienyl)dimolybdöntetrafluoroborat (I)

Umsetzung von $\left(\pi-C_{5} H_{5}\right)(C O)_{3} \mathrm{MoCH}_{3}$ mit $\mathrm{Ph}_{3} \mathrm{CBF}_{4} .0 .26 \mathrm{~g}(0.79 \mathrm{mmol}) \mathrm{Ph}_{3} \mathrm{CBF}_{4}$ werden in $10 \mathrm{ml} \mathrm{CH} \mathrm{Cl}_{2}$ gelöst und bei $-27^{\circ} \mathrm{C}$ mit $0.23 \mathrm{~g}(0.88 \mathrm{mmol})$ ( $\pi$ $\left.\mathrm{C}_{5} \mathrm{H}_{5}\right)(\mathrm{CO})_{3} \mathrm{MoCH}_{3}$ versetzt. Innerhalb von $7 \mathrm{~h}$ wird auf $+5^{\circ} \mathrm{C}$ gebracht und $6 \mathrm{~d}$ bei dieser Temperatur gehalten. Der entstandene Niederschlag wird abgetrennt und nach dem Trocknen IR-spektroskopisch als $\left[\left(\pi-\mathrm{C}_{5} \mathrm{H}_{5}\right) \mathrm{Mo}(\mathrm{CO})_{4}\right]^{+} \mathrm{BF}_{4}^{-}$identifiziert. Die Reaktionslösung wird $14 \mathrm{~d}$ bei Raumtemperatur gerührt, vom ent-

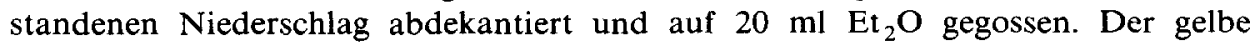
Niederschlag wird durch Zentrifugieren isoliert und $2 \mathrm{~h}$ im Hochvakuum bei Raumtemperatur getrocknet.

Umsetzung von $\left(\pi-\mathrm{C}_{5} \mathrm{H}_{5}\right)(\mathrm{CO})_{3} \mathrm{MoFBF}_{3}$ mit $\left(\pi-\mathrm{C}_{5} \mathrm{H}_{5}\right)(\mathrm{CO})_{3} \mathrm{MoCH}_{3}$. Etwa $0.36 \mathrm{~g}$

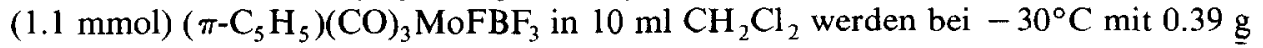


(1.5 mmol) $\left(\pi-\mathrm{C}_{5} \mathrm{H}_{5}\right)(\mathrm{CO})_{3} \mathrm{MoCH}_{3}$ versetzt und innerhalb von $18 \mathrm{~h}$ auf Raumtemperatur gebracht. Durch Zugabe von $25 \mathrm{ml}$ Hexan lässt sich ein orangefarbener Niederschlag fällen, der durch Abdekantieren von der überstehenden Lösung getrennt wird. Es werden $20 \mathrm{ml} \mathrm{CH} \mathrm{Cl}_{2}$ zugegeben. Nach einstündigem Rühren wird die Lösung vom Rückstand abpipettiert und mit Hexan versetzt, bis eine Trübung auftritt. Nach $2 \mathrm{~d}$ im Kühlschrank wird der entstandene rote Niederschlag mit der $\mathrm{CH}_{2} \mathrm{Cl}_{2}$-unlöslichen Fraktion vereinigt und im Hochvakuum $3.5 \mathrm{~h}$ getrocknet. Aus der $\mathrm{CH}_{2} \mathrm{Cl}_{2} / \mathrm{Hexan}$-Lösung scheiden sich nach mehreren Wochen bei Raumtemperatur rote Kristalle $a b$, die für die Röntgenstrukturanalyse verwendet werden. (Gef: $\mathrm{C}, 33.1 ; \mathrm{H}, 2.41 ; \mathrm{C}_{16} \mathrm{H}_{13} \mathrm{BF}_{4} \mathrm{Mo}_{2} \mathrm{O}_{5}$ ber.: $\mathrm{C}, 34.1 ; \mathrm{H}, 2.32 \%$. Molmasse 563.95)

$\mu$-Acetyl-O-(tricarbonylcyclopentadienylmolybdän)-C-(dicarbonyl-cyclopentadienyleisen) hexafluoroantimonat (II)

(a) $0.40 \mathrm{~g}$ (1.82 mmol) Acetyldicarbonylcyclopentadienyleisen und $0.83 \mathrm{~g}$ (1.73 $\mathrm{mmol})\left(\pi-\mathrm{C}_{5} \mathrm{H}_{5}\right)(\mathrm{CO})_{3} \mathrm{MoFSbF}_{5}$ werden in $10 \mathrm{ml} \mathrm{CH}_{2} \mathrm{Cl}_{2}$ bei $-25^{\circ} \mathrm{C}$ gelöst. Es tritt sofort Rotfärbung ein und ein Niederschlag fällt aus, der nach 90 min isoliert wird. Nach dem Waschen mit zweimal $10 \mathrm{ml}$ Ether wird $5.5 \mathrm{~h}$ im Hochvakuum getrocknet. Die vereinigten Waschflüssigkeiten werden zur Reaktionslösung gegeben. Der nach $15 \mathrm{~h}$ bei $-78^{\circ} \mathrm{C}$ entstehende, kristalline, dunkelrote Niederschlag wird isoliert und $4 \mathrm{~h}$ im Hochvakuum getrocknet, wobei ein gelbes Produkt absublimiert.

Aus einer Lösung von II in $\mathrm{CD}_{2} \mathrm{Cl}_{2}$ fallen nach einigen Tagen rote Kristalle aus, die für eine Röntgenstrukturanalyse verwendet werden. (Gef.: C, 31.2; II, 2.15; $\mathrm{C}_{17} \mathrm{H}_{13} \mathrm{~F}_{6} \mathrm{FeMoO}_{6} \mathrm{Sb}$ ber.: C, 29.1; H, 1.87. Molmasse 700.81)

(b) $0.48 \mathrm{~g}(1.0 \mathrm{mmol})\left(\pi-\mathrm{C}_{5} \mathrm{H}_{5}\right)(\mathrm{CO})_{3} \mathrm{MoFSbF}_{5}$ werden in $20 \mathrm{ml} \mathrm{CH}_{2} \mathrm{Cl}_{2}$ bei $-30^{\circ} \mathrm{C}$ mit $0.21 \mathrm{~g}(1.1 \mathrm{mmol})\left(\pi-\mathrm{C}_{5} \mathrm{H}_{5}\right)(\mathrm{CO})_{2} \mathrm{FeCH}_{3}$ versetzt und innerhalb $24 \mathrm{~h}$ auf Raumtemperatur gebracht und $2 \mathrm{~d}$ bei dieser Temperatur gerührt. Der entstandene Niederschlag, der $\left[\left(\pi-\mathrm{C}_{5} \mathrm{H}_{5}\right)(\mathrm{CO})_{3} \mathrm{Fe}\right]^{+} \mathrm{SbF}_{6}{ }^{-},\left\{\left[\left(\pi-\mathrm{C}_{5} \mathrm{H}_{5}\right)(\mathrm{CO})_{3} \mathrm{Mo}\right]_{2}(\mu-\mathrm{H})\right\}^{+}$ $\mathrm{SbF}_{6}^{--}$und eine weitere unbekannte Verbindung enthält, wird abgetrennt. Zur Reaktionslösung werden $30 \mathrm{ml}$-Hexan gegeben und auf $-30^{\circ} \mathrm{C}$ gekühlt. Der dabei entstehende Niederschlag (II) wird nach $40 \mathrm{~min}$ isoliert und $30 \mathrm{~min}$ im Hochvakuum getrocknet. Die verbleibende Reaktionslösung enthält ein Gemisch aus ( $\pi$ $\left.\mathrm{C}_{5} \mathrm{H}_{5}\right)(\mathrm{CO})_{3} \mathrm{MoCH}_{3}$ und $\left(\pi \sim \mathrm{C}_{5} \mathrm{H}_{5}\right)(\mathrm{CO})_{2} \mathrm{FeCOCH}_{3}$.

$\mu$-Acetyl-O-(tricarbonylcyclopentadienylmolybdïn)-C-(tetracarbonyltriphenylphosphanmangan) tetrafluoroborat (III)

Etwa $0.30 \mathrm{~g}(0.9 \mathrm{mmol})\left(\pi-\mathrm{C}_{5} \mathrm{H}_{5}\right)(\mathrm{CO})_{3} \mathrm{MoFBF}_{3}$ in $10 \mathrm{ml} \mathrm{CH}_{2} \mathrm{Cl}_{2}$ werden bei $-35^{\circ} \mathrm{C}$ mit $0.45 \mathrm{~g}(0.95 \mathrm{mmol})$ Acetyltetracarbonyltriphenylphosphanmangan verset7.t. Nach einigen Minuten tritt Verfärbung nach orangebraun ein. Die Reaktionslösung wird nach $2 \mathrm{~h}$ mit $15 \mathrm{ml}$ Hexan versetzt. Das nach $15 \mathrm{~h}$ bei $-78^{\circ} \mathrm{C}$ entstandene Öl wird isoliert, mit $20 \mathrm{ml}$ Hexan nach $3 \mathrm{~h}$ bei -35 bis $-15^{\circ} \mathrm{C}$ zur Kristallisation gebracht und $2.5 \mathrm{~h}$ im Hochvakuum getrocknet. (Gef.: $\mathrm{C}, 46.8 ; \mathrm{H}$, $2.90 ; \mathrm{C}_{32} \mathrm{H}_{23} \mathrm{BF}_{4} \mathrm{MnMoO}_{8} \mathrm{P}$ ber.: C, 47.8; H, 2.88\%. Molmasse 804.17)

\section{Röntgenstrukturanalyse von I}

Triklin, $a$ 1006.3(4), $b=1364.7(6), c=1514.7(5) \mathrm{pm}, \alpha$ 96.20(3), $\beta$ 87.45(3), $\gamma$ $115.46(3)^{\circ}, V 1867 \times 10^{6} \mathrm{pm}^{3}$; Raumgruppe $P \overrightarrow{1}, Z=4, d$ (ber.) $2.00 \mathrm{~g} \mathrm{~cm}^{-3}$, $d$ (exp.) $2.0 \mathrm{~g} \mathrm{~cm}^{-3}$. Auf einem Vierkreisdiffraktometer Syntex P2, wurden 5858 unabhängige Reflexe $\left(2^{\circ} \leqslant 2 \theta \leqslant 48^{\circ}\right)$ gemessen (Mo- $K_{\bar{\alpha}}$, Graphit-Monochromator, $\lambda 71.069 \mathrm{pm}$ ). 
TABELLE 2

ATOMPARAMETER VON I. Der anisotrope Temperaturfaktor ist definiert: $T=\exp \left[-1 / 4\left(h^{2} a^{\star 2} B_{11}\right.\right.$ $\left.\left.+k^{2} b^{\star 2} B_{22}+l^{2} c^{\star 2} B_{33}+2 h k a^{\star} b^{\star} B_{12}+2 h l a^{\star} c^{\star} B_{13}+2 k l b^{\star} c^{\star} B_{23}\right)\right] ;\left(B_{i j}\right.$ in $\left.10^{4} \mathrm{pm}^{2}\right)$

\begin{tabular}{|c|c|c|c|c|}
\hline Atom & $x / a$ & $y / b$ & $z / c$ & $B_{\text {iso }}$ \\
\hline $\operatorname{Mo}(1)$ & $-0.4335(1)$ & $-0.2933(1)$ & $-0.3880(1)$ & \\
\hline $\operatorname{Mo}(2)$ & $-0.7290(1)$ & $-0.4202(1)$ & $-0.3240(1)$ & \\
\hline $\operatorname{Mo}(3)$ & $-0.7089(1)$ & $0.0854(1)$ & $-0.2032(1)$ & \\
\hline Mo(4) & $-0.4261(1)$ & $0.2112(1)$ & $-0.1150(1)$ & \\
\hline$O(10)$ & $-0.548(1)$ & $-0.459(1)$ & $-0.358(1)$ & $0.4(2)$ \\
\hline$C(10)$ & $-0.519(2)$ & $-0.389(1)$ & $-0.283(1)$ & $7.7(3)$ \\
\hline$C(12)$ & $-0.241(3)$ & $-0.302(2)$ & $-0.346(2)$ & $13.1(7)$ \\
\hline$O(12)$ & $-0.141(2)$ & $-0.306(1)$ & $-0.331(1)$ & $3.3(3)$ \\
\hline$C(13)$ & $-0.384(2)$ & $-0.172(2)$ & $-0.287(1)$ & $10.0(5)$ \\
\hline$O(13)$ & $-0.355(2)$ & $-0.104(1)$ & $-0.233(1)$ & $3.8(3)$ \\
\hline$C(14)$ & $-0.401(2)$ & $-0.153(1)$ & $-0.471(1)$ & $9.1(4)$ \\
\hline$C(15)$ & $-0.518(2)$ & $-0.242(1)$ & $-0.511(1)$ & $8.6(4)$ \\
\hline$C(16)$ & $-0.471(2)$ & $-0.318(2)$ & $-0.542(1)$ & $11.3(5)$ \\
\hline$C(17)$ & $-0.320(2)$ & $-0.279(2)$ & $-0.522(1)$ & $9.9(5)$ \\
\hline$C(18)$ & $-0.273(2)$ & $-0.173(1)$ & $-0.482(1)$ & $9.4(4)$ \\
\hline$C(11)$ & $-0.464(2)$ & $-0.412(2)$ & $-0.198(1)$ & $10.2(5)$ \\
\hline$C(21)$ & $-0.709(2)$ & $-0.267(2)$ & $-0.320(1)$ & $11.4(5)$ \\
\hline$O(21)$ & $-0.709(2)$ & $-0.183(1)$ & $-0.316(1)$ & $3.4(3)$ \\
\hline$C(22)$ & $-0.789(2)$ & $-0.444(1)$ & $-0.456(1)$ & $9.4(4)$ \\
\hline $\mathrm{O}(22)$ & $-0.825(2)$ & $-0.465(1)$ & $-0.525(1)$ & $3.5(3)$ \\
\hline$C(24)$ & $-0.975(2)$ & $-0.472(1)$ & $-0.283(1)$ & $9.0(4)$ \\
\hline$C(25)$ & $-0.959(2)$ & $-0.568(1)$ & $-0.309(1)$ & $8.5(4)$ \\
\hline$C(26)$ & $-0.855(2)$ & $-0.575(2)$ & $-0.256(1)$ & $11.4(5)$ \\
\hline$C(27)$ & $-0.816(2)$ & $-0.488(2)$ & $-0.187(1)$ & $10.6(5)$ \\
\hline$C(28)$ & $-0.886(2)$ & $-0.424(2)$ & $-0.206(1)$ & $10.7(5)$ \\
\hline$O(30)$ & $-0.567(1)$ & $0.042(1)$ & $-0.130(1)$ & $0.5(2)$ \\
\hline$C(30)$ & $-0.632(2)$ & $0.093(1)$ & $-0.075(1)$ & $7.6(3)$ \\
\hline$C(32)$ & $-0.628(2)$ & $0.235(1)$ & $-0.232(1)$ & $8.5(4)$ \\
\hline$O(32)$ & $-0.595(1)$ & $0.323(1)$ & $-0.256(1)$ & $1.8(2)$ \\
\hline$C(33)$ & $-0.599(2)$ & $0.057(2)$ & $-0.314(1)$ & $9.8(4)$ \\
\hline$O(33)$ & $-0.543(1)$ & $0.035(1)$ & $-0.374(1)$ & $1.8(2)$ \\
\hline $\mathrm{C}(34)$ & $-0.943(2)$ & $-0.060(2)$ & $-0.172(1)$ & $10.0(5)$ \\
\hline$C(35)$ & $-0.922(2)$ & $-0.053(2)$ & $-0.272(1)$ & $10.9(5)$ \\
\hline$C(36)$ & $-0.916(2)$ & $0.055(1)$ & $-0.285(1)$ & $8.9(4)$ \\
\hline$C(37)$ & $-0.923(2)$ & $0.113(1)$ & $-0,205(1)$ & $9.5(4)$ \\
\hline$C(38)$ & $-0.939(2)$ & $0.037(2)$ & $-0.136(1)$ & $4.3(4)$ \\
\hline$C(31)$ & $-0.704(2)$ & $0.051(2)$ & $0.009(1)$ & $9.8(4)$ \\
\hline$C(41)$ & $-0.385(2)$ & $0.200(1)$ & $0.012(1)$ & $9.6(4)$ \\
\hline$O(41)$ & $-0.357(2)$ & $0.198(1)$ & $0.084(1)$ & $2.6(3)$ \\
\hline$C(42)$ & $-0.495(2)$ & $0.322(1)$ & $-0.058(1)$ & $3.5(3)$ \\
\hline$O(42)$ & $-0.526(2)$ & $0.389(1)$ & $-0.029(1)$ & $3.0(3)$ \\
\hline$C(44)$ & $-0.252(2)$ & $0.199(1)$ & $-0.223(1)$ & $9.0(4)$ \\
\hline$C(45)$ & $-0.301(2)$ & $0.269(1)$ & $-0.252(1)$ & $8.5(4)$ \\
\hline$C(46)$ & $-0.264(2)$ & $0.361(1)$ & $-0.182(1)$ & $9.2(4)$ \\
\hline$C(47)$ & $-0.189(2)$ & $0.343(1)$ & $-0.114(1)$ & $8.8(4)$ \\
\hline$C(48)$ & $-0.182(2)$ & $0.241(1)$ & $-0.141(1)$ & $9.2(4)$ \\
\hline$F(11)$ & $0.004(2)$ & $0.800(2)$ & $0.397(1)$ & \\
\hline$F(12)$ & $0.098(2)$ & $0.911(2)$ & $0.527(1)$ & \\
\hline$F(13)$ & $0.235(3)$ & $0.835(4)$ & $0.446(2)$ & \\
\hline$F(14)$ & $0.058(7)$ & $0.746(3)$ & $0.503(3)$ & \\
\hline $\mathrm{B}(1)$ & $0.114(4)$ & $0.846(3)$ & $0.471(3)$ & $17.0(11)$ \\
\hline
\end{tabular}


TABELLE 2 (Fortsetzung)

\begin{tabular}{|c|c|c|c|c|c|c|}
\hline Atom & \multicolumn{2}{|c|}{$x / a$} & $y / b$ & $z / c$ & $B_{\mathrm{i} \varkappa}$ & \\
\hline$F(21)$ & \multicolumn{2}{|c|}{$0.963(2)$} & $0.252(2)$ & $0.049(1)$ & & \\
\hline$F(22)$ & \multicolumn{2}{|c|}{$1.143(3)$} & $0.326(3)$ & $-0.055(2)$ & & \\
\hline$F(23)$ & \multicolumn{2}{|c|}{$1.181(3)$} & $0.248(2)$ & $0.058(2)$ & & \\
\hline$F(24)$ & \multicolumn{2}{|c|}{$1.171(3)$} & $0.398(3)$ & $0.095(3)$ & & \\
\hline $\mathrm{B}(2)$ & \multicolumn{2}{|c|}{$1.123(5)$} & $0,312(4)$ & $0.032(3)$ & \multicolumn{2}{|l|}{$22.0(16)$} \\
\hline$H(14)$ & \multicolumn{2}{|c|}{$\begin{array}{r}-0.3959 \\
-0.6180\end{array}$} & -0.0879 & -0.4342 & \multicolumn{2}{|l|}{5.0} \\
\hline $\mathrm{H}(15)$ & & & -0.2459 & -0.5194 & \multicolumn{2}{|l|}{5.0} \\
\hline $\mathrm{H}(16)$ & \multicolumn{2}{|c|}{-0.5202} & -0.3809 & -0.5848 & \multicolumn{2}{|l|}{5.0} \\
\hline $\mathrm{H}(17)$ & \multicolumn{2}{|c|}{-0.2546} & -0.3201 & -0.5225 & \multicolumn{2}{|l|}{5.0} \\
\hline $\mathrm{H}(18)$ & \multicolumn{2}{|c|}{-0.1648} & -0.1097 & -0.4704 & \multicolumn{2}{|l|}{5.0} \\
\hline$H(24)$ & \multicolumn{2}{|c|}{$\begin{array}{r}-1.0444 \\
1.0104\end{array}$} & -0.4542 & -0.3071 & \multicolumn{2}{|l|}{50} \\
\hline$H(25)$ & & & 0.6222 & 0.3578 & \multicolumn{2}{|l|}{5.0} \\
\hline$H(26)$ & \multicolumn{2}{|c|}{$\begin{array}{r}1.0104 \\
-0.7982\end{array}$} & -0.6166 & -0.2528 & \multicolumn{2}{|l|}{5.0} \\
\hline $\mathrm{H}(27)$ & \multicolumn{2}{|c|}{-0.7407} & -0.4656 & -0.1317 & \multicolumn{2}{|l|}{5.0} \\
\hline $\mathrm{H}(28)$ & \multicolumn{2}{|c|}{-0.8802} & -0.3529 & -0.1621 & \multicolumn{2}{|l|}{5.0} \\
\hline$H(34)$ & \multicolumn{2}{|c|}{-0.9557} & -0.1202 & -0.1404 & 5.0 & \\
\hline$H(35)$ & & & -0.1127 & -0.3062 & 5.0 & \\
\hline $\mathrm{H}(36)$ & & & 0.0793 & -0.3426 & 5.0 & \\
\hline $\mathrm{H}(37)$ & & & 0.1882 & -0.1844 & 5.0 & \\
\hline$H(38)$ & & & 0.0499 & -0.0685 & 5.0 & \\
\hline $\mathrm{H}(44)$ & & & 0.1210 & -0.2482 & 5.0 & \\
\hline$H(45)$ & & & 0.2644 & -0.3173 & 5.0 & \\
\hline$H(46)$ & & & 0.4251 & -0.1842 & 5.0 & \\
\hline$H(47)$ & & & 0.3973 & -0.0586 & 5.0 & \\
\hline $\mathrm{H}(48)$ & & & 0.2091 & -0.1066 & 5.0 & \\
\hline Atom & $B_{11}$ & $B_{22}$ & $B_{33}$ & $B_{12}$ & $B_{13}$ & $B_{23}$ \\
\hline Mo(1) & $2.61(5)$ & $2.44(5)$ & $2.90(6)$ & $1.11(4)$ & $0.31(4)$ & $0.37(4)$ \\
\hline $\operatorname{Mo}(2)$ & $2.55(5)$ & $2.39(5)$ & $2.82(6)$ & $0.91(4)$ & $0.21(4)$ & $0.37(4)$ \\
\hline $\operatorname{Mo}(3)$ & $2.31(5)$ & $2.46(5)$ & $2.81(5)$ & $1.03(4)$ & $0.36(4)$ & $0.27(4)$ \\
\hline $\operatorname{Mo}(4)$ & $2.36(5)$ & $2.61(5)$ & $2.59(5)$ & $0.93(4)$ & $0.43(4)$ & $0.27(4)$ \\
\hline$F(11)$ & $3.4(9)$ & $4.6(10)$ & $4.9(10)$ & $3.4(8)$ & $-2.8(8)$ & $-1.9(8)$ \\
\hline$F(12)$ & $5.5(11)$ & $4.7(10)$ & $1.2(7)$ & $2.1(9)$ & $1.2(7)$ & $-2.1(7)$ \\
\hline$F(13)$ & $5.5(14)$ & $26.7(45)$ & $5.2(14)$ & $12.0(24)$ & $0.0(11)$ & $-2,9(19)$ \\
\hline$F(14)$ & $28.7(61)$ & $4.1(15)$ & $10.7(28)$ & $4.8(24)$ & $-12.0(36)$ & $0.3(15)$ \\
\hline$F(21)$ & $0.2(5)$ & $7.8(13)$ & $3.2(8)$ & $-0.2(7)$ & $-0.8(5)$ & $2.2(9)$ \\
\hline$F(22)$ & $3.5(10)$ & $11.5(21)$ & $3.4(10)$ & $2.7(12)$ & $1.4(8)$ & $3,7(12)$ \\
\hline$F(23)$ & $4.6(11)$ & $4.2(11)$ & $9.3(17)$ & $3.3(10)$ & $-0.2(11)$ & $1.5(11)$ \\
\hline$F(24)$ & $6.3(15)$ & $7.8(18)$ & $13.3(26)$ & $4.4(14)$ & $-6.4(17)$ & $-9.2(20)$ \\
\hline
\end{tabular}

Die Messdaten wurden Lorentz-, Polarisations- und einer empirischen AbsorptionsKorrektur unterworfen. Die Schweratome wurden durch MULTAN (Syntex XTL). die übrigen Nicht-Wasserstoffatome durch Differenz-Fourier-Synthesen lokalisiert. Die Wasserstoffatome wurden nach idealer Geometrie berechnet. Verfeinerung der Nicht-Wasserstoffatome nach der Methode der kleinsten Quadrate mit der vollständigen Matrix mit teilweise anisotropen, teilweise isotropen Temperatur- 
TABELLE 3

AUSGEWÄHLTE ABSTANDE (pm) UND WINKEL $\left({ }^{\circ}\right)$ IN I (Entsprechende Werte aus beiden unabhängigen Molekülen sind einander gegenübergestellt. $M(1)-(4)$ repräsentiert die Schwerpunkte der $\mathrm{C}_{5} \mathrm{H}_{5}$-Ringe)

\begin{tabular}{|c|c|c|c|}
\hline$M \circ(1)-M \circ(2)$ & $290.8(2)$ & $\operatorname{Mo}(3)-\operatorname{Mo}(4)$ & $291 .(2)$ \\
\hline$M o(1)-C(10)$ & $208.2(15)$ & $\operatorname{Mo}(3)-C(30)$ & $209.1(16)$ \\
\hline $\mathrm{Mo}(1)-\mathrm{O}(10)$ & $214.3(10)$ & $\mathrm{Mo}(3)-\mathrm{O}(30)$ & $214.2(12)$ \\
\hline $\mathrm{Mo}(1)-\mathrm{C}(12)$ & $212(3)$ & $\mathrm{Mo}(3)-\mathrm{C}(32)$ & $194(2)$ \\
\hline $\operatorname{Mo}(1)-C(13)$ & $204(2)$ & $\mathrm{Mo}(3)-\mathrm{C}(33)$ & $206(2)$ \\
\hline $\operatorname{Mo}(2)-C(10)$ & $208.1(18)$ & $\mathrm{Mo}(4)-\mathrm{C}(30)$ & $211.7(16)$ \\
\hline $\mathrm{Mo}(2)-\mathrm{O}(10)$ & $213.2(12)$ & $\mathrm{Mo}(4)-\mathrm{O}(30)$ & $211.8(10)$ \\
\hline $\operatorname{Mo}(2)-C(21)$ & $201(2)$ & $\operatorname{Mo}(4)-C(41)$ & $203(2)$ \\
\hline $\mathrm{Mo}(2)-\mathrm{C}(22)$ & $207(2)$ & $\mathrm{Mo}(4)-\mathrm{C}(42)$ & $201(2)$ \\
\hline$C(10)-O(10)$ & $136(2)$ & $C(30)-O(30)$ & $136(2)$ \\
\hline$C(10)-C(11)$ & $153(3)$ & $C(30)-C(31)$ & $150(2)$ \\
\hline $\operatorname{Mo}(1)-M(1)$ & 200 & $\mathrm{Mo}(3)-\mathrm{M}(3)$ & 200 \\
\hline $\operatorname{Mo}(2)-M(2)$ & 199 & $\operatorname{Mo}(4)-M(4)$ & 201 \\
\hline $\operatorname{Mo}(2)-\operatorname{Mo}(1)-C(10)$ & $45.7(5)$ & $\operatorname{Mo}(3)-\operatorname{Mo}(4)-C(30)$ & $47.3(3)$ \\
\hline $\mathrm{Mo}(2)-\mathrm{Mo}(1)-\mathrm{O}(10)$ & $47.0(3)$ & $\operatorname{Mo}(3)-\operatorname{Mo}(4)-\mathrm{O}(30)$ & $45.9(4)$ \\
\hline $\mathrm{Mo}(2)-\mathrm{Mo}(1)-\mathrm{C}(12)$ & $124.6(7)$ & $\operatorname{Mo}(3)-\operatorname{Mo}(4)-\mathrm{C}(41)$ & $124.1(6)$ \\
\hline $\mathrm{Mo}(2)-\mathrm{Mo}(1)-\mathrm{C}(13)$ & $94.3(6)$ & $\mathrm{Mo}(3)-\mathrm{Mo}(4)-\mathrm{C}(42)$ & $91.3(5)$ \\
\hline $\mathrm{Mo}(2)-\mathrm{Mo}(1)-\mathrm{M}(1)$ & 122.3 & $\operatorname{Mo}(3)-M o(4)-M(4)$ & 122.6 \\
\hline$M(1)-M o(1)-C(10)$ & 165.0 & $M(4)-M o(4)-C(30)$ & 161.4 \\
\hline$C(10)-\operatorname{Mo}(1)-C(12)$ & $79.8(8)$ & $C(30)-M o(4)-C(41)$ & $78.3(7)$ \\
\hline $\mathrm{C}(10)-\mathrm{Mo}(1)-\mathrm{C}(13)$ & $81.3(7)$ & $C(30)-M o(4)-C(42)$ & $85.1(7)$ \\
\hline $\mathrm{O}(10)-\mathrm{Mo}(1)-\mathrm{C}(12)$ & $85.9(8)$ & $\mathrm{O}(30)-\mathrm{Mo}(4)-\mathrm{C}(41)$ & $90.3(6)$ \\
\hline $\mathrm{O}(10)-\mathrm{Mo}(1)-\mathrm{C}(13)$ & $118.8(6)$ & $\mathrm{O}(30)-\mathrm{Mo}(4)-\mathrm{C}(42)$ & $121.9(6)$ \\
\hline $\mathrm{C}(12)-\mathrm{Mo}(1)-\mathrm{C}(13)$ & $84.1(9)$ & $C(41)-M o(4)-C(42)$ & $83.0(8)$ \\
\hline $\mathrm{Mo}(1)-\mathrm{Mo}(2)-\mathrm{C}(10)$ & $47.3(3)$ & $\mathrm{Mo}(4)-\mathrm{Mo}(3)-\mathrm{C}(30)$ & $46.6(4)$ \\
\hline $\mathrm{Mo}(1)-\mathrm{Mo}(2)-\mathrm{O}(10)$ & $45.7(5)$ & $\operatorname{Mo}(4)-\operatorname{Mo}(3)-\mathrm{O}(30)$ & $46.6(3)$ \\
\hline $\mathrm{Mo}(1)-\mathrm{Mo}(2)-\mathrm{C}(21)$ & $74.6(7)$ & $\mathrm{Mo}(4)-\mathrm{Mo}(3)-\mathrm{C}(32)$ & $70.2(5)$ \\
\hline $\mathrm{Mo}(1)-\mathrm{Mo}(2)-\mathrm{C}(22)$ & $84.7(5)$ & $\operatorname{Mo}(4)-\operatorname{Mo}(3)-C(33)$ & $98.0(6)$ \\
\hline $\mathrm{Mo}(1)-\mathrm{Mo}(2)-\mathrm{M}(2)$ & 163.0 & $\operatorname{Mo}(4)-\operatorname{Mo}(3)-\mathrm{M}(3)$ & 155.0 \\
\hline $\mathrm{M}(2)-\mathrm{Mo}(2)-\mathrm{C}(10)$ & 117.8 & $\mathrm{M}(3)-\mathrm{Mo}(3)-\mathrm{C}(30)$ & 114.1 \\
\hline$C(10)-M o(2)-C(21)$ & $99.9(8)$ & $\mathrm{C}(30)-\mathrm{Mo}(3)-\mathrm{C}(32)$ & $105.3(7)$ \\
\hline $\mathrm{C}(10)-\mathrm{Mo}(2)-\mathrm{C}(22)$ & $123.0(7)$ & $\mathrm{C}(30)-\mathrm{Mo}(3)-\mathrm{C}(33)$ & $121.3(7)$ \\
\hline $\mathrm{O}(10)-\mathrm{Mo}(2)-\mathrm{C}(21)$ & $121.7(7)$ & $\mathrm{O}(30)-\mathrm{Mo}(3)-\mathrm{C}(32)$ & $116.2(6)$ \\
\hline $\mathrm{O}(10)-\mathrm{Mo}(2)-\mathrm{C}(22)$ & $90.6(6)$ & $\mathrm{O}(30)-\mathrm{Mo}(3)-\mathrm{C}(33)$ & $85.5(6)$ \\
\hline$C(21)-M o(2)-C(22)$ & $88.9(9)$ & $\mathrm{C}(32)-\mathrm{Mo}(3)-\mathrm{C}(33)$ & $86.1(8)$ \\
\hline$O(10)-C(10)-C(11)$ & $121.4(14)$ & $\mathrm{O}(30)-\mathrm{C}(30)-\mathrm{C}(3 \mathrm{l})$ & $121.9(14)$ \\
\hline $\operatorname{Mo}(1)-C(10)-\operatorname{Mo}(2)$ & $88.6(6)$ & $\operatorname{Mo}(3)-C(30)-\operatorname{Mo}(4)$ & $87.6(6)$ \\
\hline $\operatorname{Mo}(1)-\mathrm{O}(10)-\mathrm{Mo}(2)$ & $85.7(4)$ & $\mathrm{Mo}(3)-\mathrm{O}(30)-\mathrm{Mo}(4)$ & $86.2(4)$ \\
\hline
\end{tabular}

faktoren (Wasserstoffatome wurden nicht verfeinert; Atomformfaktoren für ungeladene Atome 1t. International Tables) konvergierte gegen $R-0.093$ und $R_{n}=$ 0.111 für 4887 Strukturfaktoren $\left(F_{0} \geqslant 5 \sigma\right)$. Die Atomparameter sind in Tab. 2, ausgewählte Abstände und Winkel in Tab. 3 wiedergegeben.

\section{Dank}

Der Deutschen Forschungsgemeinschaft und dem Fonds der Chemischen Industrie gilt unser Dank für die Förderung unserer Arbeiten. 


\section{Literatur}

1 X. Mitteilung: W. Beck, K. Raab, U. Nagel und M. Steimann, Angew. Chem., im Druck.

2 W. Beck und K. Schloter, Z. Naturforsch. B, 33 (1978) 1214.

3 B.W. Hames und P. Legzdins, Organometallics, 1 (1982) 116.

4 L.M. Venanzi, Coord. Chem. Rev., 43 (1982) 251.

5 (a) B. Olgemöller und W. Beck, Chem. Ber., 114 (1981) 2360; (b) R. Usón, J. Forniés. P. Espinet. R. Navarro und M.A. Usón, Inorg. Chim. Acta, 33 (1979)L 103; (c) G. Hartmann, R. Froböse. R. Mews und G.M. Sheldrick, Z. Naturforsch. B, Im Druck.

6 Bei der Umsetzung von $\left(\pi-\mathrm{C}_{5} \mathrm{H}_{5}\right)(\mathrm{CO})_{3} \mathrm{MoFBF}_{3}$ mit $\left(\pi-\mathrm{C}_{5} \mathrm{H}_{5}\right) \mathrm{Mo}(\mathrm{CO})_{3} \mathrm{CH}_{3}$ wurde zunächst ein methylverbrückter Komplex angenommen: W. Beck, K. Schloter und H. Ernst, abstracts IX. Internat. Conference on Organomettalic Chemistry, C 53, September 1979. Dijon.

7 S.J. LaCroce und A.R. Cutler, J. Amer. Chem. Soc., 104 (1982) 2312.

8 S.B. Butts, S.H. Strauss, E.M. Holt, R.E. Stimson, N.W. Alcock und D.F. Shriver, J. Amer. Chem. Soc., 102 (1980) 5093; J.P. Collman, R. Finke, J.N. Cawse und J.I. Brauman, J. Amer. Chem. Soc., 100 (1978) 4766.

9 U. Nagel, K. Sünkel und W. Beck, unveröffentlicht.

10 Vgl., z.B. E.O. Fischer und V. Kiener, J. Organometal. Chem., 23 (1970) 215; A. Mayr, Y.C. Lin, N.M. Boag und H.D. Kaesz, Inorg. Chem., 21 (1982) 1704: G. Erker. K. Kropp, C. Krüger und An-Pei Chiang, Chem. Ber. 115 (1982) 2447 und dort zit. Lit.

11 M.R. Churchill und H.J. Wasserman, Inorg. Chem., 21 (1982) 226.

12 R.D. Adams, D.A. Katahira und Li-Wu Yang. Organometallics, 1 (1982) 231.

13 B.E.R. Schilling, R. Hoffmann und D.L. Lichtenberger, J. Amer. Chem. Soc., 101 (1979) 585.

14 U. Schubert, Organometallics, 1 (1982) 1085.

15 R.B. King, Organometallic Syntheses, Vol. 1, Academic Press, New York, 1965 (a) S. 145; (b) S. 151.

16 R.B. King, J. Amer. Chem. Soc, 85 (1963) 1918.

17 K. Noack, U. Ruch und F. Calderazzo, Inorg. Chem., 7 (1968) 345. 